\title{
Progressive myoclonus epilepsy associated with neuroserpin inclusion bodies (neuroserpinosis)
}

\author{
Benoit D. Roussel ${ }^{1,2}$, David A. Lomas ${ }^{1}$, \\ Damian C. Crowther ${ }^{3,4}$ \\ ${ }^{1}$ Wolfson Institute for Biomedical Research, UCL, London, UK \\ 2 Inserm U919, GIP CYCERON, University Caen Basse Normandie, Caen, France \\ 3 Department of Genetics, University of Cambridge, UK \\ ${ }^{4}$ MedImmune Limited, Cambridge, United Kingdom
}

\begin{abstract}
Familial encephalopathy with neuroserpin inclusion bodies (FENIB) is a conformational proteinopathy characterised by neuronal inclusion bodies composed of the serine protease inhibitor (SERPIN), neuroserpin. Presenting clinically as a familial dementia-epilepsy syndrome, the molecular mechanism of the pathogenic abnormalities in neuroserpin has been characterised at atomic resolution. There is a remarkable genotypephenotype correlation between the degree of molecular destabilisation of the several variants of the neuroserpin protein, their propensity to selfassociate and the age of onset of the dementia-epilepsy complex. As with other serpinopathies there appears to be a mix of cell-autonomous toxicity, due to neuronal accumulation of neuroserpin, and non-cell autonomous toxicity, caused by loss of protease inhibition, in this case the dysregulated protease is likely to be tissue plasminogen activator (tPA). FENIB should be considered in cases of progressive myoclonic epilepsy and dementia particularly where there is family history of neuropsychiatric disease.
\end{abstract}

Key words: serpins, serpinopathy, protease inhibitor, conformational disease, neuroserpin, dementia, familial progressive myoclonic epilepsy, FENIB, progressive myoclonus epilepsies

Familial encephalopathy with neuroserpin inclusions bodies (FENIB) has been identified as a cause of pre-senile dementia with frontal symptoms as well as progressive myoclonic epilepsy (Minassian et al., 2016). In affected individuals, mutated neuroserpin accumulates in neuronal inclusions (Collins bodies). FENIB is due to mutations in the SERPINI1 gene located on chromosome $3 q 26$. Serine protease inhibitors (serpins) are a large superfamily of proteins that employ a conserved molecular mechanism for the inhibition of a wide range of proteases. Mutations may render serpins prone to polymerisation, i.e. ordered aggregation, in the endoplasmic reticulum of the synthetic Acell. In the case of neuroserpin (NS), a neuronal protein, this aggregation causes gain-offunction neuronal dysfunction 
that is thought to underpin the neurological manifestations. NS also inhibits TPA and, in this way, is thought to regulate the sensitivity of neurones to glutamatergic excitatory neurotransmission at the NMDA ( $\mathrm{N}$-methyl-D-aspartate) receptor. The epileptic component of FENIB may be due to dysfunction of the NS/tPA pathway.

Neuroserpin (NS) is a member of the serine protease inhibitor, or serpin, superfamily and has sequence and structural homologies to the archetype $\alpha_{1}$-antitrypsin. At the molecular level, serpins are composed of $9 \alpha-$ helices, $3 \beta$-sheets ( $A$ to $C$ ) and an exposed mobile reactive centre loop ( $R C L$ ). The $R C L$ typically contains 20 residues that act as a pseudo-substrate for the target protease (Elliott et al., 1996; Ryu et al., 1996). The main role of NS is to regulate the plasmin proteolytic pathway, in particular by inhibiting tissue-type plasminogen activator (tPA). In this regard, NS plays physiological roles in the development of the central nervous system (Seeds et al., 1999), in learning and memory and also in such pathological events as stroke (Cole et al., 2007) and epilepsy (Qian et al., 1993).

Numerous mutations in human serpins have been linked to a wide range of diseases; examples include emphysema, angioedema, and dementia with progressive myoclonus epilepsy, resulting from mutations in $\alpha_{1}$-antitrypsin, C1-inhibitor, and neuroserpin, respectively. Direct toxicity is invariably a consequence of the intracellular accumulation of serpin aggregates that are termed polymers, and may result in death of the synthetic cell. In the case of neuroserpin, cytotoxicity is seen exclusively in neurons of the central nervous system. There may also be associated loss-of-function effects caused by the deregulated hyperactivity of the target proteases and this may underpin the development of epilepsy in patients carrying neuroserpin variants. To date, only a few families and exceptionally rare non-familial cases of progressive myoclonic epilepsy linked to the SERPINI1 gene have been described. Age at onset is between 13 and 60 years and the disorder is severe with rapid loss of autonomy and premature death.

\section{Pathophysiology of neuroserpin-related disease}

Neuroserpin was first identified as an axonallysecreted glycoprotein in the conditioned medium of embryonic chick dorsal root ganglia cells (Stoeckli et al., 1989; Osterwalder et al., 1996). In mammals it is expressed in the central and peripheral nervous system, predominantly in neurons (Hastings et al., 1997; Osterwalder et al., 1998) where it can inhibit a number of trypsin-like enzymes, including thrombin and plasmin (Osterwalder et al., 1998), however, subsequent studies indicate that PA is the main physiological target. Accordingly, the highest levels of NS are found in parts of the nervous system that have the highest expression of tPA mRNA and protein (Krueger et al., 1997).

While not all serpins interact with proteases, those such as neuroserpin, which have retained inhibitory activity, employ a highly conserved molecular mechanism. The sequence of events that results in inhibition begins with the serpin's RCL (labelled in red in figure $1 M$ ) entering the active site of the cognate protease, initially behaving as a substrate. The enzyme and serpin form a transient intermediate called the Michaelis complex (Ye et al., 2001) which precedes the cleavage of the RCL at a specific position, termed the $\mathrm{P} 1-\mathrm{P} 1$ ' bond. This leads to the release of the $\mathrm{P} 1$ residue and the formation of an ester bond between the Cterminal portion of the serpin and the protease. At this stage, however, further hydrolysis, that would otherwise release cleaved serpin and active protease, is prevented by a profound conformational change in the serpin (Wright \& Scarsdale, 1995). This transition from a "stressed to relaxed" form occurs as the proteaseattached remainder of the RCL inserts as a $\alpha$-strand in the main $\beta$-sheet $A$ of the serpin core. This insertion is highly energetically favourable and violently flips the enzyme from the upper to the lower pole of the serpin (Huntington et al., 2000), causing steric denaturation and inactivation of the protease. Permanent destruction of the protease is subsequently achieved by third-party proteolysis of those domains of the target protease that have been rendered unstructured (Huntington et al., 2000). Experimentally, the enzymeserpin complex is relatively stable with a dissociation rate constant that is an order of magnitude slower than the association rate (Belorgey et al., 2002). However, dissociation of the serpin-enzyme complex does occur, in which case the released neuroserpin is in the inactive, cleaved conformation. In contrast, when tPA is liberated from the complex, it retains its proteolytic activity(Osterwalder et al., 1998).

\section{Clinical features and genotype-phenotype correlations}

Mutations in the neuroserpin gene were first reported to cause an autosomal dominant form of pre-senile dementia (Belorgey et al., 2002), described as familial encephalopathy with neuroserpin inclusion bodies (FENIB), characterized histologically by unique neuronal inclusion bodies and biochemically by polymers of the neuron-specific serpin, neuroserpin. These authors reported 2 unrelated Caucasian families living in the United States, carrying 2 different heterozygous mutations (S49P and S52R). In the larger family, affected individuals presented clinically around the 


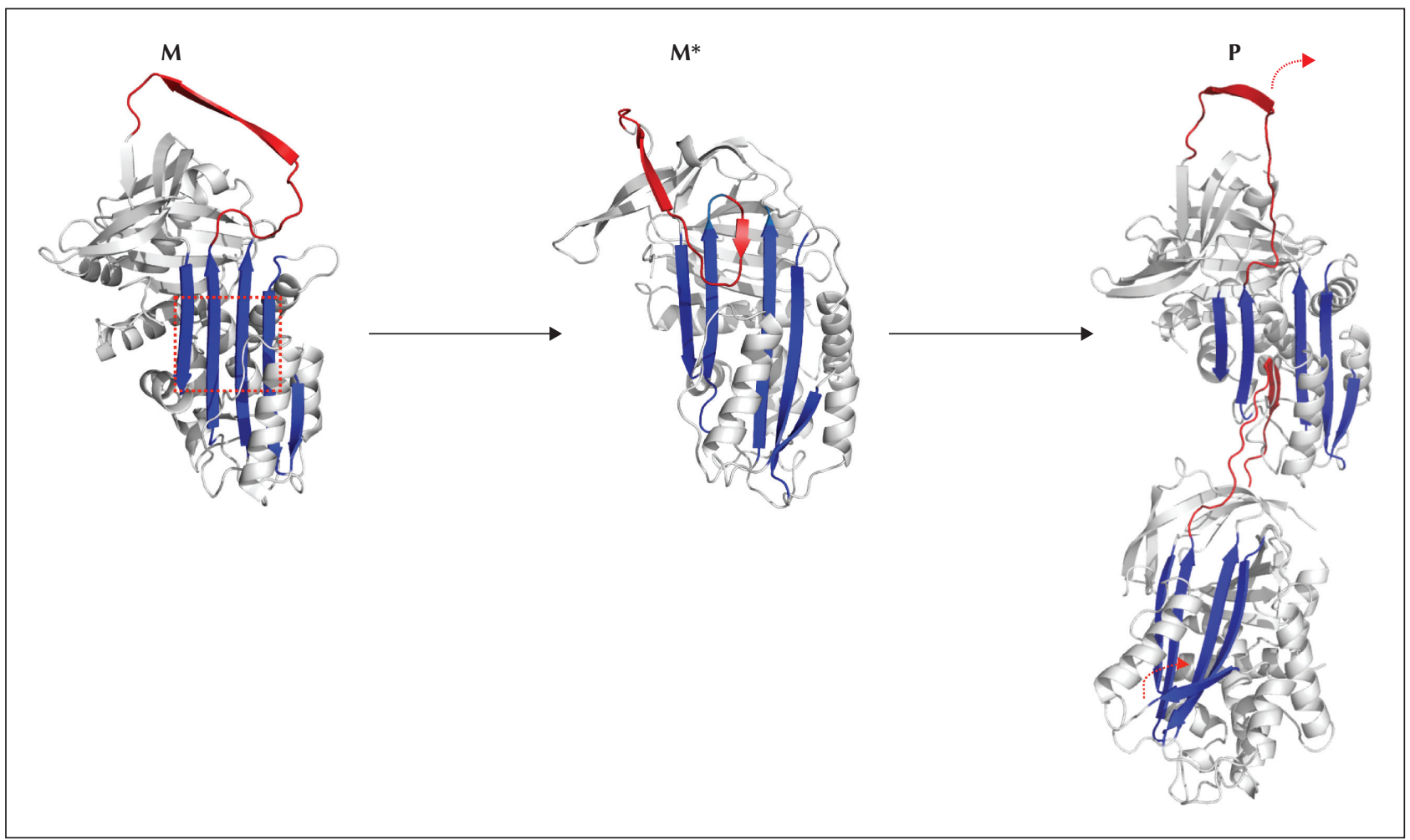

Figure 1. Molecular models of serpin conformers. $M$ represents the active protease inhibitor with the reactive loop shown in red and $\beta$-sheet A in blue. Mutations that destabilize sheet A (typically in the so-called 'shutter region' signified by the red dashed box) allow the partial insertion of the reactive loop to form a new strand in sheet $A\left(M^{*}\right)$. The consequent opening of the sheet allows the reactive loop from another serpin to insert forming a serpin dimer $(\mathrm{P})$. The repeated addition of serpins by loop-sheet polymerisation in this way results in large aggregates.

fifth decade of life with cognitive decline, including deficits in attention and concentration, response regulation difficulties, and impaired visuospatial skills. Memory was also impaired, but to a lesser degree than is typically seen in individuals with Alzheimer's disease. After several years of disease progression, most affected individuals were unable to work and eventually required nursing-home care. The second, much smaller family showed an earlier clinical onset, during the second or third decade of life. Affected individuals presented with both epilepsy and progressive cognitive decline and their neuro-histology was dominated by eosinophilic, PAS-positive intraneuronal inclusions in the brain. Later on, a small family with 2 affected siblings featuring progressive myoclonus epilepsy and dementia was described (Belorgey et al., 2002). The affected individuals developed generalized seizures in adulthood and progressed to status epilepticus over several years. In addition, they also developed slow speech, diplopia, nystagmus, dysarthria, and myoclonus in the extremities, with rapidly progressive dementia. Their deceased mother was, reportedly, similarly affected.

Davis et al. (1999a) reported additional patients with the disorder: a 23-year-old man with an 8-year history of progressive myoclonic epilepsy, dementia, tremor, and dysarthria. The second patient was a 13-year-old girl with progressive myoclonus epilepsy with intractable seizures, myoclonus, and dementia. She died at the age of 19 during status epilepticus. Her father was said to be 'mentally deficient', and a paternal uncle had died from epilepsy at the age of 18 .

Gourfinkel-An et al. (2007) subsequently described a small French family with progressive myoclonic epilepsy associated with a frontal syndrome starting from the age of 18 with severe myoclonus, generalized tonic-clonic seizures, and absences. The EEG of one of the patients showed diffuse slow waves, spikes, and spike-wave discharges superimposed on a slow background, with photic sensitivity at around $1 \mathrm{~Hz}$. Cerebral MRI revealed cortico-subcortical atrophy. The patient's condition progressively worsened and swallowing difficulties were noticed at an early stage. Additionally, cerebellar symptoms and pyramidal signs were also present. Cognitive deterioration was severe (Mini-Mental Status Examination score: 12/30). Signs of frontal dysfunction were observed (emotional lability, distractibility, and poor performance on sequential motor tests) with sparing of long-term explicit memory. In another affected relative, who died at the age of 33, 
Table 1. Various mutations in the neuroserpin gene cause onset of dementia and myoclonic epilepsy at a wide range of ages. The most aggregation-prone neuroserpin variants in vitro cause juvenile forms of FENIB while less destabilizing mutations are not penetrant until the seventh decade. Histologically, patients with early onset disease exhibit a higher burden of cortical neuroserpin inclusions (+ through to ++++$)$.

\begin{tabular}{|llll}
\hline Mutation & Onset (years) & Clinical findings & Inclusions \\
\hline S49P & $45-63$ & Dementia and terminal seizures & + \\
\hline S52R & $20-40$ & Myoclonus, Dementia & ++ \\
\hline L47P & $20-30$ & Progressive myoclonus epilepsy & +++ \\
\hline H338R & 15 & Progressive myoclonus epilepsy & +++ \\
\hline G392E & 13 & Progressive myoclonus epilepsy, chorea & +++ \\
\hline G392R & 8 & Epilepsy with slow wave sleep \\
\hline
\end{tabular}

symptoms were similar and were first noticed when she was 18. Behavioural problems, depression, and frontal dysfunction were noticed. Epilepsy was drugresistant and she experienced, during the course of the disorder, two episodes of status epilepticus. She became mute and bedridden and died of inhalation pneumonia at the age of 33. In these patients, a heterozygous S52R missense mutation at position 273 in exon 2 was detected, the same as in the two families from the United States.

A few non-familial cases have been also described. Coutelier et al. (2008) reported an 11-year-old girl who had had normal development until 8 years of age, when she developed a rapidly-progressive symptomatology, including aggressive behaviour, intellectual decline, psychic seizures, and subtle seizures with eyelid myoclonus. The EEG was suggestive of epilepsy with continuous spike-and-waves during slow-wave sleep. This patient was the first person identified with the G392R mutation in neuroserpin that resulted in severe juvenile phenotype and had accordingly appeared de novo. Hagen et al. (2011) more recently reported an additional sporadic patient with progressive myoclonus epilepsy and declining mental status starting in adulthood. Generalized seizures occurred early with myoclonus and progressive gait disturbances. Neuroimaging revealed mild atrophy and multiple periventricular white matter lesions, consistent with demyelination. The course was one of progressive decline with death occurring at the age of 34. Genetic analysis revealed a nucleotide substitution, resulting in a proline to leucine amino acid substitution (L47P). The genotype-phenotype correlations are remarkably strong, with mutations causing increasingly severe clinical features in the following order: S49P, S52R, L47P, H338R, G392E and G392R (table 1). In general, increasing clinical severity is characterized by earlier onset of the symptom and an increasing contribution from the epileptic component of the syndrome. More specifically, individuals with the S49P mutation have diffuse small intraneuronal inclusions of neuroserpin with an onset of dementia between the ages of 45 and 60 (Davis et al., 1999a; Davis et al., 1999b; Bradshaw et al., 2001). People with the S52R, L47P and H338R variants have larger and more numerous intraneuronal inclusions associated with progressively earlier onset of symptoms, during early adulthood (S52R, L47P) and adolescence (H338R) (Hagen et al., 2011; Miranda et al., 2008). In the most severe cases, caused by the most polymerogenic mutations, namely G392R and G392E, the patients exhibit the earliest onset of symptoms, with profound intellectual decline during childhood associated with severe, uncontrolled epilepsy (Coutelier et al., 2008). While FENIB is a rare disease, with only a few known kindreds worldwide, diagnosis should be considered in patients that present with a frontal-type dementia combined with epilepsy, particularly when eosinophilic inclusions are seen on brain biopsy or at post-mortem.

\section{The pathological polymerisation of neuroserpin variants results in FENIB}

These remarkable genotype-phenotype correlations that are evident in the clinic are mirrored by the biophysical and biochemical properties of the variant NS proteins. Under physiological conditions, the rate of aggregation of the least clinically-aggressive NS mutant, S49P, is more than 10 times higher than that of the wild type protein, whereas the association rate constant for tPA is essentially unchanged (Belorgey et al., 2002). The next most severe mutation, S52R, results in a further tenfold increase in the polymerization rate and the loss of effective tPA inhibition (Belorgey et al., 2004). Thereafter, the aggregation rate becomes so 
high as to be practically immeasurable. In the classic model of serpin polymerisation, proposed by Carrell and Lomas (2002), the functional changes in NS are all caused by the progressive destabilization of the key structural element, termed $\beta$-sheet $A$, which forms the core of NS (figure 1). This structural perturbation favours the incorporation of the RCL from a second NS molecule over the physiological process of intramolecular RCL insertion that occurs during protease inhibition (Davis et al., 2002). An initial loop-sheet polymerization event yields a NS dimer that retains a patent $\beta$-sheet $A$ at one end and a destabilized RCL at the other. Such a dimer is competent to undergo further loop-sheet polymerization to form trimers and eventually higher-order polymers. Recently, Huntington and colleagues have proposed alternative mechanisms for polymerization that require a more significant domain swap between the serpins in a chain (Sado et al., 2009; Huntington \& Whisstock, 2010; Belorgey et al., 2011; Singh \& Jairajpuri, 2011; Yamasaki et al., 2011). In either case, the NS polymers gradually become entangled in the neuronal endoplasmic reticulum (ER) and form inclusions, known as Collins bodies. This phenomenon of aggregation or 'polymerization' has been described in other serpins such as $\alpha_{1}$-antitrypsin where it results in hepatocyte inclusions and liver disease (Lomas et al., 1992; Elliott et al., 1996; Huntington et al., 1999). The observation that FENIB was caused by NS mutations (S49P and H338R) that are structurally homologous to substitutions in $\alpha_{1}$-antitrypsin, which also lead to polymerization (S53F and H334D, respectively) (Lomas et al., 1993), argues strongly in favour of a shared molecular mechanism. This was confirmed by the finding that the neuronal inclusion bodies of FENIB were formed by polymers of NS with identical morphology to the polymers of mutant $\alpha_{1}$-antitrypsin present in hepatocytes from a child with $\alpha_{1}$-antitrypsin deficiency-related cirrhosis (Davis et al., 1999a; Carrell \& Lomas, 2002).

\section{Dissecting the pathological mechanisms of the dementing and epileptic components of FENIB}

The ER-resident inclusions (Miranda et al., 2004) in the neurones of individuals expressing NS variants are likely to represent the toxic gain-of-function that results in this dominantly-inherited neurodegenerative syndrome. Serpin polymerisation exerts a stress on the cell that differs in important ways from other proteinopathies. In most cases, misfolding of proteins in the ER results in activation of the unfolded protein response (UPR). However, the native-like structure of serpin polymers results in a distinct signalling pathway called the ER overload response (Davies et al., 2009).
This ER overload response activates NF- $\mathrm{B}$ by a pathway that is dependent on raised cytoplasmic $\mathrm{Ca}^{2+}$ levels. ER-associated degradation or ERAD is involved in the degradation of mutant NS and may be able to degrade the polymers (Ying et al., 2011), whereas autophagy is more important in the bulk turnover of both wild type and mutant NS (Kroeger et al., 2009). In the presence of polymerogenic mutations in serpins, and with increasing age, these mechanisms are overwhelmed and retention of polymers in the ER leads to cell death. This is apparent in the fly model of FENIB where the accumulation of intracellular polymers is associated with locomotor deficits where there was a correlation between the severity of the behavioural deficits and the degree of polymer accumulation (Miranda et al., 2008).

While the intraneuronal accumulation of NS inclusions may underpin the dementia seen in FENIB, there is evidence that other mechanisms may also contribute to the epileptic propensity of these patients. In particular, it is notable that epilepsy is rarely seen when normal levels of tPA inhibitory activity are present, as is the case for wild type and S49P NS. This has been recently highlighted in relation to stroke, where patients thrombolyzed with recombinant IPA present a higher level of seizures (Alvarez et al., 2013). When tPA inhibition is lost (for example, for the S52R and 392 mutants) then epilepsy becomes the major clinical feature (Davis et al., 2002). Notably, neuroserpin is able to dampen neuronal sensitivity to excitotoxic stimuli by regulating tPA activity (Yepes et al., 2000; Yepes et al., 2002; Wu et al., 2010). This effect appears to be mediated by the tPAmediated proteolytic cleavage of the NR1 subunit of the NMDA receptor that increases the functioning of this excitatory glutamate receptor (figure 2) (Nicole et al., 2001; Fernandez-Monreal et al., 2004; Samson et al., 2008; Baron et al., 2010).

Excess glutamatergic neurotransmission is also a potent cause of epilepsy (reviewed in Vincent \& Mulle [2009]) and increased NMDA signalling in response to dysregulated tPA activity could contribute to FENIB. Indeed, co-injection of neuroserpin with kainate into the hippocampus of the mouse attenuated the spread of consequent epileptic activity when compared to co-injection of kainate with vehicle alone (Yepes et al., 2002). These data suggest a role for tPA in seizure spread in epilepsy and support the use of NS or others tPA inhibitors as a potential therapy.

\section{Conclusion}

Familial encephalopathy with neuroserpin inclusion bodies is a recently described neurodegenerative disease that is responsible for progressive myoclonic epilepsy and/or pre-senile dementia. Serpinopathies 


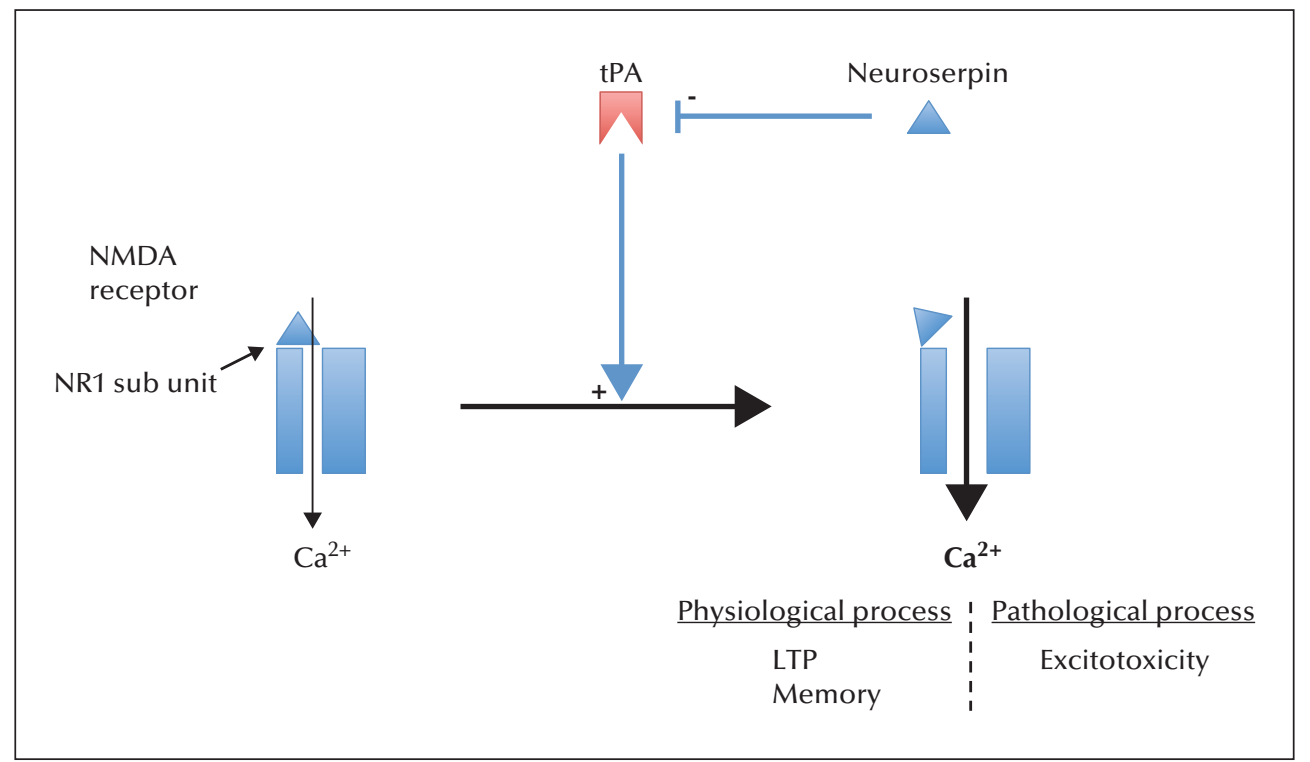

Figure 2. The NMDA receptor mediates the influx of calcium into neurones in the presence of glutamate. tPA has been shown to cleave the NR1 subunit (triangle) of the receptor and, as a consequence, increases the concentration of intracellular calcium. By inhibiting tPA, neuroserpin is thought to protect neurons from the toxic consequences of excessive NMDA activity, termed 'excitotoxicity'.

are unique among conformational diseases because they form native-like aggregates or polymers in the ER of synthetic cells. Serpin variants accumulate as inclusion bodies and thus activate the ER-overload response. It appears that this gain-of-function toxicity is responsible for the neuronal dysfunction and death that underpins dementia in FENIB. The evidence is less clear as to whether similar gain-of-toxicity is the cause of the epilepsy in FENIB or whether this results from dysfunction of the NS/tPA system. The relative importance of these two mechanisms has yet to be clearly elucidated. To date, genetic analysis of the SERPINI1 gene should be performed in patients with adult-onset PME and early-onset, rapidly progressive cognitive dysfunction or predominantly frontal dementia.

\section{Disclosures.}

None of the authors have any conflict of interest to disclose.

The present manuscript is part of a Epileptic Disorders/ Mariani Foundation supplement on Progressive Myoclonus Epilepsies, downloadable as a whole by visiting www. epilepticdisorders.com.

\section{References}

Alvarez V, Rossetti AO, Papavasileiou V, Michel P. Acute seizures in acute ischemic stroke: does thrombolysis have a role to play? J Neurol 2013; 260:55-61.

Baron A, Montagne A, Cassé F, et al. NR2D-containing NMDA receptors mediate tissue plasminogen activator-promoted neuronal excitotoxicity. Cell Death Differ 2010; 17: 860-71.
Belorgey D, Crowther DC, Mahadeva R, Lomas DA. Mutant neuroserpin (S49P) that causes familial encephalopathy with neuroserpin inclusion bodies is a poor proteinase inhibitor and readily forms polymers in vitro. J Biol Chem 2002; 277: 17367-73.

Belorgey D, Sharp LK, Crowther DC, Onda M, Johansson J, Lomas DA. Neuroserpin portland (Ser52Arg) is trapped as an inactive intermediate that rapidly forms polymers: implications for the epilepsy seen in the dementia FENIB. Eur J Biochem 2004; 271: 3360-7.

Belorgey D, Irving JA, Ekeowa UI, et al. Characterisation of serpin polymers in vitro and in vivo. Methods 2011; 53: 255-66.

Bradshaw CB, Davis RL, Shrimpton AE, et al. Cognitive deficits associated with a recently reported familial neurodegenerative disease: familial encephalopathy with neuroserpin inclusion bodies. Arch Neurol 2001; 58:1429-34.

Carrell RW, Lomas DA. Alpha1-antitrypsin deficiency - a model for conformational diseases. N Engl J Med 2002; 346: 45-53.

Cole JW, Naj AC, O'Connell JR, et al. Neuroserpin polymorphisms and stroke risk in a biracial population: the stroke prevention in young women study. BMC Neurol 2007; 7: 37.

Coutelier M, Andries S, Ghariani S, et al. Neuroserpin mutation causes electrical status epilepticus of slow-wave sleep. Neurology 2008; 71: 64-6.

Davis RL, Shrimpton AE, Holohan PD, et al. Familial dementia caused by polymerization of mutant neuroserpin. Nature 1999a; 401:376-9.

Davis RL, Holohan PD, Shrimpton AE, et al. Familial encephalopathy with neuroserpin inclusion bodies. Am J Pathol 1999b; 155: 1901-13. 
Davis RL, Shrimpton AE, Carrell RW, et al. Association between conformational mutations in neuroserpin and onset and severity of dementia. Lancet 2002; 359: 2242-7.

Davies MJ, Miranda E, Roussel BD, Kaufman RJ, Marciniak SJ, Lomas DA. Neuroserpin polymers activate NF-kappaB by a calcium signaling pathway that is independent of the unfolded protein response. J Biol Chem 2009; 284: 18202-9.

Elliott PR, Lomas DA, Carrell RW, Abrahams J-P. Inhibitory conformation of the reactive loop of $\alpha_{1}$-antitrypsin. Nat Struct Biol 1996; 3: 676-81.

Fernandez-Monreal M, López-Atalaya JP, Benchenane K, et al. Arginine 260 of the amino-terminal domain of NR1 subunit is critical for tissue-type plasminogen activator-mediated enhancement of $\mathrm{N}$-methyl-D-aspartate receptor signaling. J Biol Chem 2004; 279: 50850-6.

Gourfinkel-An I, Duyckaerts C, Camuzat A, et al. Clinical and neuropathologic study of a French family with a mutation in the neuroserpin gene. Neurology 2007; 69: 79-83.

Hagen MC, Murrell JR, Delisle MB, et al. Encephalopathy with neuroserpin inclusion bodies presenting as progressive myoclonus epilepsy and associated with a novel mutation in the proteinase inhibitor 12 gene. Brain Pathol 2011; 1: 575-82.

Hastings GA, Coleman TA, Haudenschild CC, et al. Neuroserpin, a brain-associated inhibitor of tissue plasminogen activator is localized primarily in neurons. Implications for the regulation of motor learning and neuronal survival. J Biol Chem 1997; 272: 33062-7.

Huntington JA, Whisstock JC. Molecular contortionism - on the physical limits of serpin 'loop-sheet' polymers. Biol Chem 2010; 391: 973-82.

Huntington JA, Pannu NS, Hazes B, Read RJ, Lomas DA, Carrell RW. A $26 \AA ̊$ structure of a serpin polymer and implications for conformational disease. J Mol Biol 1999; 293: 449-55.

Huntington JA, Read RJ, Carrell RW. Structure of a serpinprotease complex shows inhibition by deformation. Nature 2000; 407: 923-6.

Kroeger H, Miranda E, MacLeod I, et al. Endoplasmic reticulum-associated degradation (ERAD) and autophagy cooperate to degrade polymerogenic mutant serpins. J Biol Chem 2009; 284: 22793-802.

Krueger SR, Ghisu GP, Cinelli P, et al. Expression of neuroserpin, an inhibitor of tissue plasminogen activator, in the developing and adult nervous system of the mouse. J Neurosci 1997; 17: 8984-96.

Lomas DA, Evans DL, Finch JT, Carrell RW. The mechanism of $\mathrm{Z} \alpha_{1}$-antitrypsin accumulation in the liver. Nature 1992; 357 : 605-7.

Lomas DA, Finch JT, Seyama K, Nukiwa T, Carrell RW. $\alpha_{1}$-antitrypsin, $S_{\text {iiyama }}$ (Ser $\left.{ }^{53} \varnothing P h e\right) ; ~ f u r t h e r$ evidence for intracellular loop-sheet polymerisation. J Biol Chem 1993; 268: 15333-5.

Minassian BA, Striano P, Avanzini G. Progressive Myoclonus Epilepsies: State-of-the-Art. Epileptic Disord 2016; 18(Suppl. 2): S1-158.
Miranda E, Romisch K, Lomas DA. Mutants of neuroserpin that cause dementia accumulate as polymers within the endoplasmic reticulum. J Biol Chem 2004; 279: 28283-91.

Miranda E, MacLeod I, Davies MJ, et al. The intracellular accumulation of polymeric neuroserpin explains the severity of the dementia FENIB. Hum Mol Genet 2008; 17:1527-39.

Nicole $\mathrm{O}$, Docagne $\mathrm{F}$, Ali $\mathrm{C}$, et al. The proteolytic activity of tissue-plasminogen activator enhances NMDA receptormediated signaling. Nat Med 2001; 7:59-64.

Osterwalder T, Contartese J, Stoeckli ET, Kuhn TB, Sonderegger P. Neuroserpin, an axonally secreted serine protease inhibitor. EMBO J 1996; 15: 2944-53.

Osterwalder T, Cinelli P, Baici A, et al. The axonally secreted serine proteinase inhibitor, neuroserpin, inhibits plasminogen activators and plasmin but not thrombin. J Biol Chem 1998; 273: 2312-21.

Qian Z, Gilbert ME, Colicos MA, Kandel ER, Kuhl D. Tissueplasminogen activator is induced as an immediate-early gene during seizure, kindling and long-term potentiation. Nature 1993; 361: 453-7.

Ryu S-E, Choi H-J, Kwon K-S, Lee KN, Yu M-H. The native strains in the hydrophobic core and flexible reactive loop of a serine protease inhibitor: crystal structure of an uncleaved $\mathrm{a}_{1}$-antitrypsin at $2.7 \AA ̊$ At. Structure 1996; 4: 1181-92.

Sado M, Yamasaki Y, Iwanaga T, et al. Protective effect against Parkinson's disease-related insults through the activation of XBP1. Brain Res 2009; 1257: 16-24.

Samson AL, Nevin ST, Croucher D, et al. Tissue-type plasminogen activator requires a co-receptor to enhance NMDA receptor function. J Neurochem 2008; 107: 1091-101.

Seeds NW, Basham ME, Haffke SP. Neuronal migration is retarded in mice lacking the tissue plasminogen activator gene. Proc Natl Acad Sci U S A 1999; 96:14118-23.

Singh $\mathrm{P}$, Jairajpuri MA. Strand $6 \mathrm{~B}$ deformation and residues exposure towards N-terminal end of helix B during proteinase inhibition by serpins. Bioinformation 2011;5: 315-9.

Stoeckli ET, Lemkin PF, Kuhn TB, Ruegg MA, Heller M, Sonderegger $\mathrm{P}$. Identification of proteins secreted from axons of embryonic dorsal-root-ganglia neurons. Eur J Biochem 1989; 180: 249-58.

Vincent $\mathrm{P}$, Mulle C. Kainate receptors in epilepsy and excitotoxicity. Neuroscience 2009; 158: 309-23.

Wright HT, Scarsdale JN. Structural basis for serpin inhibitor activity. Proteins 1995; 22: 210-25.

Wu J, Echeverry R, Guzman J, Yepes M. Neuroserpin protects neurons from ischemia-induced plasmin-mediated cell death independently of tissue-type plasminogen activator inhibition. Am J Pathol 2010; 177: 2576-84.

Yamasaki M, Sendall TJ, Pearce MC, Whisstock JC, Huntington JA. Molecular basis of alpha1-antitrypsin deficiency revealed by the structure of a domain-swapped trimer. $E M B O$ Rep 2011;12:1011-7. 
Ye S, Cech AL, Belmares $\mathrm{R}$, et al. The structure of a Michaelis serpin-protease complex. Nat Struct Biol 2001; 8: 979-83.

Yepes M, Sandkvist M, Wong MK, et al. Neuroserpin reduces cerebral infarct volume and protects neurons from ischemia-induced apoptosis. Blood 2000; 96: 569-76.
Yepes M, Sandkvist M, Coleman TA, et al. Regulation of seizure spreading by neuroserpin and tissue-type plasminogen activator is plasminogen-independent. J Clin Invest 2002; 109: 1571-8.

Ying Z, Wang $H$, Fan $H$, Wang G. The endoplasmic reticulum (ER)-associated degradation system regulates aggregation and degradation of mutant neuroserpin. J Biol Chem 2011; 286: 20835-44. 